\title{
DESCARTES: LAS IDEAS Y SU FALSEDAD
}

LORENA GARCÍA

INSTITUTO DE INVESTIGACIONES FLLOSÓFICAS

UNIVERSIDAD NACIONAL AUTÓNOMA DE MÉXICO

En este artículo, propongo una interpretación de la noción de la idea en Descartes que incorpora de manera natural dos de sus tesis que usualmente se piensan en conflicto con otros aspectos importantes de su filosofía: que algunas de nuestras ideas son materialmente falsas, y que algunas ideas son oscuras y confusas. ${ }^{1}$

Algunos estudiosos de la filosofía de Descartes sostienen que su noción de la falsedad material es $o$ incoherente, o desastrosa para él. Anthony Kenny, por ejemplo, piensa que la tesis cartesiana de que existen ideas ma-

1 Usaré aquí las abreviaturas siguientes de las ediciones de las obras de Descartes y de otros autores:

AT: Oeuvres de Descartes, ed. Ch. Adam y P. Tannery, Cerf, Paris, 1897-1913; reimpresión: Vrin, Paris, 1957-1958, 12 vols.

CB: Descartes' Conversation with Burman, trad., introd. y comentario de John Cottingham, Clarendon Press, Oxford, 1976.

CSM: The Philosophical Writings of Descartes, trad. John Cottingham, Robert Stoothoff y Dugald Murdoch, Cambridge University Press, Cambridge, 1985, vols. I y ll.

CSMK: The Philosophical Writings of Descartes, trad. John Cottingham, Robert Stoothoff, Dugald Murdoch y Anthony Kenny, Cambridge University Press, Cambridge, 1991 vol. III.

Suárez DA: Francisco Suárez, Comentarios a los libros de Aristóteles sobre el alma, ed. bilingüe y trad. de Carlos Baciero y Luis Baciero, ed. crítica de Salvador Castellote, Editorial Labor, Madrid, 1981, 4 vols.

Suárez DM: Francisco Suárez, Disputaciones metafísicas, ed. bilingüe y trad. de S. Rabade Romeo, S. Caballero Sánchez y A. Puigcerver Zanon, Editorial Gredos, Madrid, 1960, 7 vols.

Aquinas DA: Thomas Aquinas, In Aristotelis librum De Anima commentarium, editado por A.M. Pirotta, 4a. ed., Marietti, 1959.

FH: Aristotle's De Anima in the Version of William of Moerbeke and the Commentary of St. Thomas Aquinas, trad. Kenelm Foster y Silvester Humphries, introd. de Ivo Thomas, Yale University Press, New Haven, 1951.

Usaré el estilo comúnmente aceptado para citar partes del texto de las ediciones de las obras de Descartes; $y$ las traducciones al inglés que se encuentran en CB, CSM y CSMK, puesto que son las traducciones estándar, a menos que indique explícitamente que la traducción es mía. Para citar a Suárez, mencionaré la obra (por ejemplo, la disputación, sección y artuculo), y el volumen y número de página en el que la cita aparece en las ediciones bilingües arriba mencionadas. Usaré las traducciones al castellano de estas ediciones. 
terialmente falsas es incoherente; ${ }^{2}$ mientras que Margaret Wilson sostiene que la noción de la falsedad material en Descartes es un desastre, ya que

it entails that the objective reality of an idea is not something the idea wears on its face. Descartes would have it otherwise. ${ }^{3}$

Wilson está en lo correcto al afirmar que esto es lo que el concepto de falsedad material implica. De hecho, el concepto de oscuridad y confusión también tiene como consecuencia que las ideas poseen un nivel de contenido que no es inmediatamente accesible al cognoscente.

La interpretación que propongo fue expuesta claramente por Descartes en su respuesta a la objeción de Antoine Arnauld, quien sostenía que no puede haber ideas materialmente falsas. Como veremos más adelante, en el marco conceptual escolástico aristotélico, un concepto no puede ni ser falso ni representar erróneamente a su objeto, puesto que, por definición, el objeto del concepto es aquella cosa con la cual el concepto se conforma. En esencia, ésta es la objeción de Arnauld a la teoría de Descartes. A esta objeción Descartes responde que una idea puede ser materialmente falsa (u oscura y confusa) porque lo que una idea parece representar puede diferir, en ciertos aspectos, de lo que una idea realmente representa. Voy a hacer ver claramente cómo es que, en el caso de una idea clara y distinta, lo que la idea parece representar (y que es el contenido de la idea que es inmediatamente accesible al cognoscente) coincide, al menos en parte, con lo que la idea realmente representa, lo cual nos permite un acceso mediato a este segundo nivel de contenido. En cambio, en una idea que no es clara y distinta, lo que la idea parece representar no necesariamente coincide

2 Anthony Kenny, Descartes: A Study of His Philosophy, Random House, Nueva York, 1968, p. 121.

3 Margaret Wilson, Descartes, Routledge and Kegan Paul, Londres, 1978, p. 112. Wilson dice que la réplica de Descartes a la objeción de Antoine Arnauld respecto a la falsedad material, es un modelo de "confusion confounded", ibid., p. 110. Martha Bolton también considera que "it would be fatal for Descartes to hold that the cognitive content of an idea can diverge from the object of the idea"; que es una tesis que aparentemente subyace en el concepto de falsedad material. Véase su artículo "Confused and Obscure Ideas of Sense", en Essays on Descartes' Meditations, ed. Amelie Oksenberg Rorty, University of California Press, Berkeley, Los Ángeles, Londres, 1986, p. 393. En un artículo reciente, Richard W. Field también intenta mostrar que la noción de falsedad material en Descartes no es ni desastrosa ni incoherente; pero su interpretación padece de ciertas inadecuaciones textuales fundamentales. Por ejemplo, ningún texto apoya la afirmación de Field de que Descartes usa 'idea considerada materialmente' en dos sentidos diferentes; o su afirmación de que una idea considerada formalmente es la idea en tanto que representa a un objeto que existe, ya que en ninguno de los pasajes en los que Descartes define esta noción se hace referencia a la existencia de los objetos de las ideas. Véase su articulo "Descartes on the Material Falsity of Ideas", Philosophical Review, no. 102, 1993, pp. 309-333; y mi artículo "Descartes on Mental Representation", manuscrito, nn. 80,82 y 84 . 
con lo que la idea representa; lo cual puede ocasionar la comisión de algún error acerca de lo que la idea representa.

Procederé de la siguiente manera: En la primera parte, introduciré brevemente algunos de los conceptos de Francisco Suárez y de Tomás de Aquino que son de capital importancia para entender la discusión que se da entre Arnauld y Descartes acerca de la posibilidad de las ideas materialmente falsas. La segunda parte presenta el concepto de idea en Descartes y las tesis que se relacionan con este concepto. La tercera parte examina en detalle la discusión entre Descartes y Arnauld acerca de la falsedad material. La cuarta parte desarrolla una reconstrucción lógica de los conceptos cartesianos de idea, claridad y distinción, oscuridad y confusión, y falsedad material; asimismo, en esta parte se muestra que esta reconstrucción se adecua a los textos de Descartes. En la quinta y última parte se responde a la objeción de Wilson de que la noción cartesiana de falsedad material es desastrosa.

\section{Suárez y Aquino}

En Aquino y Suárez, la teoría hilomórfica de la sustancia se encuentra íntimamente ligada a sus teorías del conocimiento y de la representación mental. Estos autores sostienen que la forma de una cosa debe encontrarse en la facultad cognoscitiva apropiada para que tal cosa pueda conocerse. Las formas, en tanto que se encuentran en una determinada facultad, se denominan 'especies'. ${ }^{4}$ En consecuencia, existe una relación de conformidad entre el acto cognoscitivo y su objeto. Suárez expresa de manera explícita esta consecuencia: "Es necesario que convengan entre sí representante y representado." Llamaré a la tesis de que el objeto de un concepto (o de un acto de conocimiento) debe poseer todas las propiedades que se representan en el concepto, el 'principio de conformidad'. ${ }^{6}$

Aceptar el principio de conformidad conlleva aceptar la tesis de que, en sentido estricto, los conceptos no pueden ser falsos. ${ }^{7}$

4 Véase, por ejemplo, Aquinas DA III, lect. 2, sec. 718 (FH 419); o Suárez DA V, 1, 3 (vol. II, 286); ibid., V, 4, 15 (vol. II, 364).

5 "Habere inter se convenientiam". Suárez DM IX, 1, 14 (vol. II, 181).

6 Este principio no afirma que el objeto representado debe poseer solamente las propiedades que se representan en el concepto.

7 Suárez expresa explícitamente esta consecuencia. Véase Suárez DM LX, 1, 14 (vol. II, 181). Suárez acepta que existe una falsedad impropia (improprie dicta) que puede darse en los conceptos; véanse Suárez DM IX, 1, 21 (vol. II, 187); y DM IX, 1, 22 (vol. II, 188). Suárez acepta otro tipo de falsedad aplicable a ciertos conceptos, que él llama 'falsedad cuasi-material'. Véase Suárez DM IX, 2, 4 (vol. II, 193). En mi artículo "Descartes on Mental Representation", sección II, muestro que estos dos conceptos de falsedad conceptual no pueden ser identificados con la noción cartesiana de falsedad material. 


\section{Las ideas en Descartes}

Antes de distinguir tres maneras de considerar una idea, Descartes introduce, en la tercera Meditación, la noción de idea como sigue:

Some of my thoughts are as it were the images of things (tanquam rerum imagines), and it is only in these cases that the term 'idea' is strictly appropriate. ${ }^{8}$

Las ideas, dice Descartes, son tanquam rerum imagines. Más adelante abandona el 'imagines', cuando dice que "there can be no ideas which are not as it were of things (tanquam rerum)". ${ }^{9}$ En la versión francesa de este pasaje, dice: "il n'y en peut avoir aucune [idée] qui ne nous semble representer quelque chose". ${ }^{10}$ Las ideas entonces son aquellos de nuestros pensamientos que parecen representar alguna cosa, alguna res. ${ }^{11}$

Descartes introduce una primera distinción en la. manera de considerar una idea:

When we think of them (i.e., of ideas) as representing something we are taking them not materially but formally. If, however, we were considering them not as representing this or that but simply as operations of the intellect, then it could be said that we were taking them materially. ${ }^{12}$

Cuando las ideas son consideradas materialmente, afirma Descartes, "they all appear to come from within me in the same fashion". ${ }^{13}$ Por consiguiente, una idea considerada materialmente es la idea considerada como un modo de pensar, es decir, como se nos presenta a la conciencia; mientras que la idea considerada formalmente es la idea en tanto que representa algo.

8 AT VII 37, CSM II 25.

9 AT VII 44, CSM II 30.

10 AT IX-1 34-35. Descartes autorizó la versión francesa.

11 Véase Norman Wells, "Material Falsity in Descartes, Arnauld, and Suárez", Journal of the History of Philosophy, no. 22, 1984, pp. 25-50, en donde él sostiene que 'tanquam rerum' no debe entenderse como 'como si de cosas' o 'parece representar, ibid., p. 28 n. 20. Por tanto, de acuerdo con Wells, todas las ideas son rerum, acerca de cosas, es decir, todas las ideas representan alguna res y no sollo parecen representar. Por tanto, las ideas materialmente falsas, que "represent non-things as things (non rem tanquam rem repraesentant)", AT VII 43, CSM II 30, son pseudorepresentaciones o pseudoideas, de acuerdo con Wells, puesto que estas representan, no cosas (res), sino lo contrario de cosas, non res. Véase su "Material Falsity", p. 37. Más adelante veremos que esta interpretación es incorrecta. Véanse nn. 27 y 42.

12 AT VII 232, CSM II 163. Véase también AT VIIIA 11, CSM I 198.

${ }^{13}$ AT VII 40, CSM II 27-28. Debe tenerse cuidado de no confundir el concepto de falsedad material con el de una idea considerada materialmente. La falsedad material se denomina asi para distinguirla de la falsedad en sentido estricto (proprie dicta) o falsedad formal que es aplicable solamente a los juicios, y no a las ideas o conceptos. Una idea considerada materialmente es la idea considerada como se nos presenta a la conciencia, y esto no tiene ninguna relación interesante con el concepto de falsedad material. 
Descartes hace una segunda distinción entre una idea considerada materialmente y la idea considerada objetivamente:

'Idea' can be taken materially, as an operation of the intellect, in which case it cannot be said to be more perfect than me. Alternatively, it can be taken objectively, as the thing represented by that operation. ${ }^{14}$

Hay, por tanto, tres diferentes maneras de considerar una idea: (a) materialmente, como un modo de pensar que se nos presenta a la conciencia y que es un producto de nuestras mentes finitas; (b) formalmente, en tanto que representa algo, y (c) objetivamente, como el objeto representado. ${ }^{15}$ Examinemos cada una de éstas en mayor detalle.

\section{(A) Ideas consideradas objetivamente}

En un sentido primario, las ideas representan esencias, de acuerdo con Descartes. Esto es lo que le dice a Gassendi:

An idea represents the essence of a thing; and if anything is added to or taken away from the essence, then the idea automatically becomes the idea of something else. ${ }^{16}$

Considerada objetivamente, por tanto, la idea es una esencia (en tanto que existe objetivamente en el intelecto), ${ }^{17}$ puesto que la esencia es el objeto representado. ${ }^{18}$ Además, la esencia contenida en la idea determina el grado de perfección objetiva contenida en la idea, ${ }^{19}$ es decir, el grado de realidad objetiva de la idea. ${ }^{20}$

14 "Pro re [... .] repraesentata". AT VII 8, CSM II 7.

15 Esta distinción tripartita no es una distinción entre tres diferentes clases de ideas, sino entre tres diferentes maneras de considerar la misma idea. Véase Monte Cook, "The Alleged Ambiguity of 'Idea' in Descartes' Philosophy", The Southwestern Journal of Philosophy, no. 6, 1975, pp. 87-94. Acerca de la distinción entre idea considerada formalmente e idea considerada objetivamente, véase Norman Wells, "Objective Reality of Ideas in Descartes, Caterus, and Suárez", Journal of the History of Philosophy, no. 28, 1990, pp. 33-61, esp. 34-35.

16 AT VII 371, CSM II 256. Véase también AT VII 64-65, CSM II 44-45. El pasaje en AT VII 8 , CSM II 7 es sugerente.

17 Véase AT VII 102-103, CSM II 74-75.

18 Véase AT IV 350, CSMK 281. Una idea representa una cosa (o cosas) en tanto que representa su esencia.

19 Véase AT VII 8, CSM II 7.

20 Véanse los siguientes pasajes en los que Descartes usa 'perfección' entendida como 'realidad': AT VII 40, CSM II 28; AT VII 161, CSM II 113; AT VIILA 11, CSM I 198, y AT I 561, CSMK 86. Acerca de la noción de grados de realidad objetiva, véanse AT VII 41, CSM II 28, y AT VII 165, CSM II 117. Sobre la conexión entre realidad y esencia, véase la discusión con Burman en AT V 160, CSMK 343. 
En cambio, las ideas materialmente falsas son un caso límite, puesto que "representan no-cosas (non res) como cosas (res)". ${ }^{21}$ La versión francesa contiene una explicación del término 'non res':

j'ignore [...] si les idées que je conçois de ces qualites (comme les couleurs, les sons, les odeurs, et les saveurs) sont en effet les idées de quelques choses réelles, ou bien si elles ne me representent que des êtres chimeriques qui ne peuvent exister. ${ }^{22}$

Una idea materialmente falsa representa algo que no puede existir; algo que no es real. ${ }^{23}$ Por ende, estas ideas carecen de realidad objetiva, ${ }^{24}$ y no contienen objetivamente ninguna esencia, es decir, no representan ninguna esencia. Por el contrario, las ideas verdaderas -entre las cuales se cuentan las ideas claras y distintas- representan objetos posibles ${ }^{25}$ y esencias verdaderas e inmutables; ${ }^{26}$ mientras que ciertas ideas oscuras y confusas que uno ha construido, como las ideas de las quimeras o de las sirenas, representan tan sólo unas esencias inventadas por el intelecto. ${ }^{27}$

Hay otro aspecto importante de las ideas consideradas objetivamente; a saber, que, de acuerdo con Descartes, una idea puede contener objetivamente -o implícitamente-_ ${ }^{28}$ muchas perfecciones, o propiedades positivas, que ignoramos. Por ejemplo, Descartes le escribía a Gassendi que:

Once the idea of the true God has been conceived, although we may detect additional perfections in him which we had not yet noticed, this does not mean that we have augmented the idea of God; we have simply made it more distinct and explicit, since, so long as we suppose that our original idea was a true one, it must have contained all these perfections. ${ }^{29}$

21 AT VII 43, CSM II 30, traducción mía.

22 AT IX-134. Véanse también las notas 27 y 42.

23 Véanse AT VII 44, CSM II 30; y AT VII 234, CSM II 163.

24 Véase AT VII 44, CSM II 30.

25 Véanse AT VII 152, CSM II 108; AT V 160, CSMK 343, y la carta a Mersenne, en la que Descartes dice: "We can conceive - you should add 'distinctly' - only things which are possible and true." (AT III 215, CSMK 155.) Véanse también Margaret Wilson, Descartes, p. 108; Calvin Normore, "Meaning and Objective Being: Descartes and His Sources", en Essays on Descartes' Meditations, p. 238; y Wells, "Objective Reality", pp. 48 y 56.

26 Véanse AT VII 64, CSM II 45; AT V 160, CSMK 343, y AT V 354, CSMK 376.

27 Véase AT VI 117, CSM II 83-84. Acerca de la posición de Suárez con respecto a la categoría ontológica de las quimeras y otros entes imposibles, como un hombre irracional o un triángulo cuadrado, véase J.P. Doyle, "Suárez on Beings of Reason and Truth (1)", Vivarium, no. 25, 1987, pp. 47-72; esp. 53-60 y 69-75. Las quimeras o entes imposibles, que Suárez clasifica como 'entes de razón' (entia rationis), no tienen una realidad aparte del ser pensado. Véase Suárez DM LIV, 1-3.

28 Véase AT III 383, CSMK 184.

29 AT VII 373, CSM II 256, el subrayado es mío. Véanse también: AT VII 147, CSM II 105, y AT VIILA 24, CSM I 209. 
Las ideas oscuras y confusas son un caso extremo, ya que, consideradas por sí solas, con frecuencia uno no puede determinar qué es lo que representan - es decir, qué objeto o esencia la idea contiene objetiva o implícitamente. ${ }^{30}$

\section{(B) Ideas consideradas material y formalmente}

Descartes afirma que, entre las ideas, consideradas materialmente, "no reconozco ninguna desigualdad". ${ }^{31}$ En los Principios de la Filosofía modifica este enunciado, y dice que nuestras ideas, "in so far as they are merely modes of thinking, do not differ much from one another."32

Muchos distinguidos historiadores opinan que considerar una idea materialmente, es considerarla sin hacer referencia a su contenido. ${ }^{33}$ Sin embargo, esta interpretación, en primer lugar, carece de apoyo textual. ${ }^{34}$ En segundo lugar, la interpretación asume que una idea representa algo simplemente en virtud de su contenido. Esta presuposición, como veremos en la próxima sección, es errónea. La teoría de la representación en Descartes es mucho más compleja; de acuerdo con esta teoría, el considerar una idea materialmente es considerarla como un modo de pensar, es decir, como aquello que es inmediatamente accesible a la conciencia. Veremos que una idea considerada de esta manera es la idea en tanto que es tanquam rerum; es decir, en tanto que parece representar algo. Esto es diferente, tanto de la idea considerada objetivamente (a saber, la idea considerada como aquello que realmente se representa), como de la idea considerada formalmente (es decir, la idea en tanto que representa, o considerada en su función representativa). ${ }^{35}$ Examinaremos ahora la controversia entre Arnauld y Descartes acerca de la falsedad material, para ver cómo Descartes usa esas distinciones de manera crucial en sus argumentos.

30 Véanse AT VII 233-234, CSM II 163-164; AT VII 147, CSM II 105; y AT VII 43-44, CSM II 30.

31 "Non agnosco ullam inter ipsas inaequalitatem." AT VII 40, CSM II 27, traducción mía.

32 "Non multum a se mutuo diferre." AT VIILA 11, CSM 1198.

33 Véanse Wells, "Objective Reality", pp. 45-46; Thomas Lennon, "The Inherence Pattern and Descartes' Ideas", Journal of the History of Philosophy, no. 12, 1974, pp. 43-52, esp. 47; E.J. Ashworth, "Descartes' Theory", p. 335; y Richard W. Field, "Descartes on the Material Falsity", p. 322.

34 El único texto que quizá podría sostenerla es aquel en el que Descartes dice que, entre las ideas consideradas materialmente, no reconoce ninguna desigualdad (AT VII 40, CSM II 27). Pero, como ya hemos visto, Descartes modifica después esta afirmación, y dice que las ideas, consideradas materialmente, no difieren mucho unas de otras (AT VIILA 11, CSM I 198).

35 Véanse la n. 12 y Wells, "Objective Reality", p. 34, n. 3. 
3. Descartes y Arnauld: La falsedad material y la oscuridad de las ideas

La controversia entre estos filósofos se inicia en la tercera Meditación, en la cual Descartes define la falsedad material como aquello que ocurre en las ideas "cuando representan no-cosas como cosas". ${ }^{36}$ Por ejemplo, dice Descartes,

since there can be no ideas which are not as it were of things (tanquam rerum), if it is true that cold is nothing but the absence of heat, the idea which represents it to me as something real and positive deserves to be called false. ${ }^{37}$

Si el frío es la ausencia de una propiedad positiva (es decir, una negación), ${ }^{38}$ entonces la idea que lo representa como algo positivo ${ }^{39}$ es falsa.

La objeción de Arnauld consiste en argumentar que ninguna idea puede ser materialmente falsa. Arnauld dice:

if cold is an absence, it cannot exist objectively in the intellect by means of an idea whose objective existence is a positive entity. Therefore, if cold is merely an absence, there cannot ever be a positive idea of it, and hence there cannot be an idea which is materially false. ${ }^{40}$

Arnauld entonces ofrece un argumento muy parecido al que Suárez usa para sostener que los conceptos no pueden ser falsos, puesto que no puede haber disconformidad entre la idea representante y lo representado. Dice Arnauld:

What does the idea of cold, which you say is materially false, represent to your mind? An absence? But in that case it is true. A positive entity? But in that case it is not the idea of cold (if cold is a negation). ${ }^{41}$

Nótese que la corrección del argumento de Arnauld no depende de la verdad de la suposición de que el frío es la ausencia del calor. El argumento es acerca de cualquier idea que representa una non res. (algo que no es real,

36 AT VII 43, CSM II 30, traducción mía.

37 AT VII 44, CSM II 30.

38 Tanto las negaciones como las privaciones y los entes imposibles son, para los escolásticos aristotélicos, entidades de razón. Véase n. 27.

39 Es decir, como algo real y no simplemente como la negación de algo real.

40 AT VII 207, CSM II 145.

41 AT VII 207, CSM II 146. 
ya sea una negación o una cosa imposible) como si fuera una res. ${ }^{42}$ Veamos ahora la respuesta de Descartes.

Al comentario de Arnauld de que "if cold is merely an absence, then there cannot be an idea of cold which represents it to me as a positive thing,"43 Descartes responde: "It is clear that he [i.e., Arnauld] is dealing solely with an idea taken in the formal sense." ${ }^{44}$ Descartes nos está diciendo que el principio de conformidad -en el que Arnauld se apoya para afirmar que una idea que representa una propiedad positiva no puede ser una idea del frío (si el frío es una negación) - es aplicable sólo a la idea considerada formalmente.

Descartes procede a hacer la distinción, arriba mencionada, entre una idea considerada formalmente y la idea considerada materialmente, dando a entender que Arnauld confunde estas dos en su objeción. Cuando las ideas son consideradas materialmente, dice Descartes, "they have no reference to the truth or falsity of their objects". ${ }^{45}$ Es decir, cuando una idea se considera materialmente, la pregunta acerca de la realidad o irrealidad del objeto representado - la pregunta acerca de la categoría ontológica de este objetoestá fuera de lugar. Considerada materialmente, la idea del frío es del frío independientemente de si el frío es o una entidad positiva o una negativa. En este sentido, Descartes afirma:

whether cold is a positive thing or an absence, I do not, for that reason, have any other idea of it, but remains the same as that which I have always had. ${ }^{46}$

Así, el principio de conformidad es inaplicable a una idea considerada materialmente. Lo que el frío de hecho sea no afecta la idea que tengo del frío considerada materialmente; pero esto no sería posible si el principio de conformidad fuese aplicable, ya que, si esto fuera el caso, lo que el frío es -ya sea algo positivo o algo negativo- afectaría el carácter de la idea considerada materialmente. En consecuencia, es necesario que una idea se conforme a su objeto cuando la idea se considera no materialmente, sino formalmente; es decir, cuando la idea se considera en tanto que representa algo.

El siguiente paso en la respuesta de Descartes consiste en introducir otra distinción, y de una manera muy explícita nos dice:

42 De hecho, de acuerdo con Descartes, todas las ideas de las cualidades sensibles -incluyendo las ideas de frío y de calor - representan cosas que no son reales (AT VII 233-24, CSM II 163); es decir, entidades quiméricas que no pueden existir (AT DX-1 34-35). Nótese que una non res en Descartes corresponde a lo que tradicionalmente se denominaba una 'entidad de razón'. Véase la n. 27.

43 AT VII 206, CSM II 145.

44 AT VII 232, CSM II 163.

45 Ibid.

46 Ibid., traducción mía. 
I think we need to make a distinction: [...] for it often happens in the case of obscure and confused ideas - and the ideas of heat and cold fall into this category - that an idea is referred to something other than that of which it is in fact the idea. ${ }^{47}$

Algo muy diferente sucede en el caso de las ideas claras y distintas. Por ejemplo, la idea de Dios

cannot be said to refer to something with which it does not correspond (conformis). ${ }^{48}$

Así, una idea puede referirse a algo diferente a aquello a lo que la idea se conforma. Esto sucede en el caso de muchas ideas oscuras y confusas, y en todas las ideas materialmente falsas. Descartes se da cuenta de que una distinción como ésta es necesaria para articular las nociones de falsedad material y de oscuridad y confusión.

La distinción entre aquello a lo que la idea se refiere y aquello a lo que ésta se conforma, corresponde (respectivamente) a la distinción entre la idea considerada materialmente y la idea considerada formalmente. Una idea considerada materialmente se refiere a algo; pero esto no sería posible si el considerar una idea materialmente fuese equivalente a considerarla sin hacer referencia a su contenido. ${ }^{49}$ Debe haber un sentido en el cual es apropiado decir que, considerada materialmente, la idea, por lo menos, parece ser acerca de algo.

Para elucidar más claramente lo que, según yo, Descartes está proponiendo, ofreceré una reconstrucción lógica de los conceptos cartesianos de idea, claridad y distinción, oscuridad y confusión, falsedad material, etc. Como veremos más adelante, esta reconstrucción también nos ayuda a entender las relaciones que existen entre estos conceptos y otras tesis cartesianas.

\section{Una reconstrucción lógica}

La siguiente reconstrucción captura algunas de las relaciones lógicas que, de acuerdo con Descartes, se dan entre los conceptos de idea, esencia, claridad y distinción, oscuridad y confusión, falsedad material, etc.

47 AT VII 233, CSM II 163: "Ut ad aliud quid referantur quam ad id cujus revera ideae sunt."

48 Ibid.: "Non potest ipsam referri ad aliquid cui non sit conformis." El uso de 'conformare' en este pasaje no es un accidente; como tampoco es un accidente el que, si unimos esta cita con la anterior obtenemos lo siguiente: aquello con lo que una idea se conforma es aquello de lo cual la idea realmente es. Descartes no está rechazando el principio de conformidad; sino que sostiene que conformarse a algo no es sino uno de otros aspectos de una idea. Veremos que conformarse corresponde sólo a la idea considerada formalmente.

49 Véanse las nn. 33 y 34. 
Las versiones formalizadas de estos conceptos requieren, por supuesto, una cierta interpretación, ya que, por sí solas, estas versiones no capturan todas las connotaciones y las consecuencias de los conceptos que Descartes usa. Indicaremos los detalles de esta interpretación en los lugares apropiados.

Usaré terminología de la lógica de primer orden, así como expresiones que cuantifican sobre propiedades, lógica modal proposicional y lógica modal cuantificacional. Como es usual, ' $\square p$ ' significará, 'es necesario que $p$ ' y ‘ $\diamond p$ ' significará 'es posible que $p$ '. Debido a que Descartes acepta ciertas verdades necesarias que no son verdades lógicas, ' $\square p$ ' no puede entenderse exclusivamente como 'es lógicamente necesario que $p$ '.

\section{(A) La noción de la idea}

Consideraré que una idea es una estructura que consiste en dos conjuntos de propiedades (en tanto que tienen existencia objetiva en el intelecto), ${ }^{50}$ y de una función entre estos conjuntos. Los conjuntos que constituyen una idea $I$ se llamarán ' $M A T_{I}$ ' (la idea $I$ considerada materialmente) y ' $O B J_{I}$ ' (la idea $I$ considerada objetivamente), y la función entre estos conjuntos se llamará 'FORM'.

Cualquier conjunto finito y no vacío de propiedades puede ser un conjunto $M A T_{I}$. Interpretaremos $M A T_{I}$ como la idea $I$ en tanto que contiene aquellas propiedades que parecen estar representadas por la idea. Además, $M A T_{I}$ es aquel aspecto de la idea $I$ que es inmediatemente accesible al cognoscente; es decir, aquel contenido que está presente a la conciencia. En principio, cualquier propiedad puede pertenecer al conjunto $M A T$, ya que, de acuerdo con Descartes, una idea considerada materialmente es la idea considerada como un producto de nuestras mentes finitas y falibles. Considerada así, no hay necesidad de postular ninguna otra causa de la idea más que la habilidad de nuestras mentes de concebir y combinar contenidos de ciertas maneras que no están necesariamente de acuerdo con los principios de la representación correcta; principios que, como veremos más adelante, constituyen la función FORM y que gobiernan la representación de una cosa por medio de sus propiedades esenciales. Por ejemplo, es posible que todas las propiedades en $M A T_{I}$ sean meramente accidentales.

La relación FORM entre $M A T_{I}$ y $O B J_{I}$ es la siguiente: Si $M A T_{I}$ contiene las propiedades $P_{1}, \ldots, P_{m}$ (y ninguna otra), entonces, para toda $i$, una propiedad $Q_{i}$ pertenece a $O B J_{I}$ si y sólo si se cumplen las siguientes tres condiciones:

50 Para abreviar mi exposición, usaré, de aquí en adelante, la palabra 'propiedad' en vez de 'propiedad en tanto que tiene existencia objetiva en la mente'. Véase la n. 17. 
(a) Es necesario que cualquier cosa que posea la propiedad $Q_{i}$, la posea necesariamente; es decir,

$$
\square(\forall x)\left(Q_{i} x \supset \square Q_{i} x\right) .
$$

(b) Es necesario que cualquier cosa que posea todas las propiedades contenidas en $M A T_{I}$, posea la propiedad $Q_{i}$ necesariamente; es decir,

$$
\square(\forall x)\left[\left(P_{1} x \& \ldots \& P_{m} x\right) \supset \square Q_{i} x\right] .
$$

(c) Es posible que exista algo que posea todas las propiedades que pertenecen a $M A T_{1}$; es decir,

$$
\diamond(\exists x)\left(P_{1} x \& \ldots \& P_{m} x\right) .
$$

En sentido estricto, consideraré a FORM como una relación cuyo dominio es un conjunto de conjuntos, finitos y no vacíos, de propiedades (todos aquellos conjuntos MAT de cada una de nuestras ideas), y cuyo rango incluye el conjunto vacío y otros conjuntos de propiedades (los conjuntos $O B J$ ).

Interpretaremos $O B J_{I}$ como aquello que la idea $I$ representa, es decir, el contenido implícito de la idea. Aunque este contenido no sea inmediatamente accesible a la conciencia - como lo es $M A T_{I}$ - podemos tener acceso a $O B J_{I}$ a través de FORM, si razonamos en concordancia con las condiciones que definen esta relación. Más adelante tendremos ocasión de ampliar nuestros comentarios acerca de nuestra capacidad para razonar de esta manera.

Intuitivamente, FORM es una relación "esencializante" que toma un conjunto de propiedades (que pueden o no ser poseídas por algo esencialmente) en su dominio (p. ej., $M A T_{I}$ ), y le asigna, o bien el conjunto vacío (cuando las propiedades en $M A T_{I}$ no pueden todas ser poseídas por algo), o bien (cuando las propiedades en $M A T_{I}$ pueden todas ser poseídas por algo) el conjunto de todas y solamente aquellas propiedades que (1) sólo pueden ser poseídas esencialmente, y (2) son poseídas esencialmente por cualquier cosa que posee (esencialmente o no) todas las propiedades en $M A T_{I}$. Ahora bien, es fácil demostrar que FORM es una función. ${ }^{51}$

(B) Ideas claras y distintas

Si $M A T_{I}$ contiene las propiedades $P_{1}, \ldots, P_{m}$, y ninguna otra, entonces la idea $I$ es clara y distinta si y sólo si:

51 Véase mi artículo "Descartes on Mental Representation", sección VI. La relación inversa de FORM no es una función. Ibid. 
(a) Es posible que exista algo que posea cada una de las propiedades en $M A T_{I}$ necesariamente; es decir,

$$
\diamond(\exists x)\left(\square P_{1} x \& \ldots \& \square P_{m} x\right)
$$

(b) para cada $P_{i}$ en $M A T_{I}(i=1, \ldots, m)$, es necesario que cualquier cosa que posea esta propiedad, la posea necesariamente; es decir,

$$
\square(\forall x)\left(P_{i} x \supset \square P_{i} x\right) \quad \text { (para cada i). }
$$

Nótese que, en el caso de una idea clara y distinta $I$, todas las propiedades de $M A T_{I}$ cumplen las condiciones (a), (b) y (c) de FORM, OBJ $J_{I}$ no es el conjunto vacío; y $M A T_{I}$ es un subconjunto de $O B J_{I}$. En nuestra interpretación, esto significa que lo que una idea parece representar es, de hecho, parte de lo que la idea realmente representa, o lo que está contenido en $O B J_{l}$. Metafóricamente hablando, diríamos que la idea es transparente, ya que el aspecto de la idea que es inmediatamente accesible al cognoscente (lo contenido en $M A T_{I}$ ) coincide, al menos en parte, con lo que la idea realmente representa, esto es, con lo contenido en $O B J_{I}$. Así, considerada objetivamente, la idea está "presente y es accessible a la mente", 52 aunque posiblemente sólo de manera parcial.

Una idea clara y distinta es aquella que, en el nivel MAT, caracteriza algo por medio de propiedades que sólo pueden ser poseídas esencialmente, y es un corolario de las definiciones previas que una idea clara y distinta $I$ es tal que todas las propiedades que pertenecen a $M A T_{I}$ pueden ser poseídas por alguna cosa. Esto es lo que Descartes dice de la idea clara y distinta de Dios:

even if we conceive of God in an inadequate or, [...] 'utterly inadequate' way, this does not prevent its being certain that his nature is possible, or not selfcontradictory. ${ }^{53}$

También vimos que una idea clara y distinta es aquella que "cannot be said to refer to something with which it does not correspond". ${ }^{54}$ En nuestra reconstrucción esto corresponde al hecho de que, en una idea clara y distinta, su conjunto MAT es un subconjunto de $O B J$.

Nuestra interpretación también se confirma con la famosa discusión de Descartes en la segunda Meditación acerca de un pedazo de cera. Primero

52 Véase AT VIIL 22, CSM I 207-208, traducción mía.

53 AT VII 152, CSM II 108. Descartes dice a Mersenne: 'You quote as an axiom of mine: 'Whatever we clearly conceive is or exists.' That is not at all what I think, but only that whatever we perceive clearly is true, and so it exists if we perceive that it cannot exist; or that it can exist if we perceive that its existence is possible." Véase AT III 544-545, CSMK 211.

54 AT VII 233, CSM II 163. 
la cera se concibe por medio de sus propiedades perceptibles, tales como su tamaño y forma particulares, su color, fragancia, etc. Después de que la cera se derrite, y todas estas propiedades particulares y accidentales cambian, Descartes se pregunta:

So what was it in the wax that I understood with such distinctness? Evidently none of the features which I arrived at by means of the senses. ${ }^{55}$

\section{Descartes añade:}

the wax was not after all the sweetness of the honey, or the fragrance of the flower or $[\ldots]$ the shape. .6

Un pedazo de cera, un cuerpo, dice Descartes, es "merely something extended, flexible and changeable". ${ }^{57}$ Esta idea de la cera, que incluye sólo las propiedades esenciales de la cera, es clara y distinta, mientras que la idea previa que incluía sus propiedades accidentales (tales como su tamaño y forma particulares), es oscura y confusa, como se sigue de nuestro análisis. 58

Ahora bien, la función FORM describe, de cierta forma, la manera en la cual podemos, y debemos, formar ideas en el nivel MAT. Pero el conjunto OBJ está, por definición, constituido necesariamente por una aplicación infalible y consistente de la función FORM - algo que, por ejemplo, sólo Dios podría hacer. Es decir, que una idea considerada objetivamente está constituida por la aplicación infalible de una facultad que poseemos pero que no siempre usamos de manera consistente o infalible. Somos, de manera no-contingente, seres finitos. ${ }^{59}$

Ahora bien, cuando el conjunto MAT de una idea está constituido de manera tal que obedece los principios que constituyen la función FORM

55 AT VII 30, CSM II 20.

56 Ibid.

57 AT VII 31, CSM II 21. Véase Wilson, Descartes, p. 91, acerca del pasaje de la cera en la segunda Meditacion: "The objection he [Descartes] wishes to deal with is that to perceive the sensible modes [or properties] is the same thing as to perceive the body, in its true nature." Además, lo que Descartes está tratando de demostrar en este pasaje es "the conclusion required (as he sees it) for his physics: that it is the nature of any body at all (just) to be something extended, flexible and movable." Ibid., p. 92.

58 Ibid. Véase también el pasaje que está en AT VIIIA 30-31, CSM I 215, que demuestra la conexión entre claridad y distinción, y la esencia.

59 Véase AT VI 62, CSM II 43. Alan Gewirth expresa de manera diferente nuestra tesis de que el conjunto $O B J$ de una idea está necesariamente constituido por una aplicación consistente e infalible de la facultad FORM. Dice, en su "Clearness and Distinctness", pp. 25-26, que las ideas son "viewed normatively" cuando son vistas "from the standpoint solely of what they 'really' represent", -es decir, desde el punto de vista de lo que Descartes llama 'idea considerada objetivamente', y lo que Gewirth llama el 'contenido directo' de una idea (y que se distingue del contenido interpretativo de la idea). 
-es decir, cuando usamos la facultad FORM correctamente-, entonces la idea es clara y distinta. Algo parecido le dice Descartes a Hyperaspistes:

It is through a real faculty of the mind that it perceives two things, one apart from the other, as complete things; and [...] it is through a lack of the same faculty that the mind apprehends these two things in a confused manner, as a single thing. ${ }^{60}$

Puesto que la percepción de una cosa, como una cosa completa, incluye la percepción (o idea) clara y distinta de esa cosa, ${ }^{61} \mathrm{y}$ dado que la mente puede percibir una cosa completa sólo a través de una cierta facultad mental, se sigue que nuestra posesión de esta facultad (que llamamos FORM) hace posible que tengamos ideas claras y distintas.

\section{(C) Ideas oscuras y confusas}

Si $M A T_{I}$ contiene las propiedades $P_{1}, \ldots, P_{m}$, y ninguna otra, entonces la idea $I$ es oscura y confusa si y sólo si:

(a) no es posible que exista algo que posea cada una de las propiedades en $M A T_{I}$ necesariamente; es decir,

$$
\sim \diamond(\exists x)\left(\square P_{1} x \& \ldots \& \square P_{m} x\right)
$$

(b) existe al menos una $P_{i}$ que pertenece a $\operatorname{MAT}_{l},(i=1, \ldots, m)$, tal que es posible que exista una cosa que no posea $P_{i}$ necesariamente; es decir,

$$
\diamond(\exists x) \sim \square P_{i} x \quad \text { (para alguna } i \text { ). }
$$

Puede mostrarse fácilmente que, en mi reconstrucción, una idea oscura y confusa $I$ no puede ser clara y distinta; y que $M A T_{I}$ no es necesariamente un subconjunto de $O B J_{I}$. Además, es posible que exista una idea oscura y confusa $I$ tal que ninguna de las propiedades en $M A T_{I}$ pertenezcan a $O B J_{I}$; es decir, es posible que, el conjunto MAT de algunas ideas oscuras y confusas no contenga ninguna propiedad que cumpla con las condiciones (a) y (b) de FORM.

Por ejemplo, la idea original del pedazo de cera que lo caracterizaba (en el nivel MAT) por medio de propiedades no-esenciales (su tamaño y forma particulares) era oscura y confusa, de acuerdo con Descartes. Nuestro análisis coincide: En este caso, $M A T_{l}$ contiene sólo propiedades accidentales.

60 AT III 434, CSMK 197.

61 Véase la discusión con Amauld acerca de la noción de una cosa completa y su conexión con las ideas claras y distintas, en AT VII 220-227, CSM II 155-160. 
$O B J_{I}$ no contiene ninguna de ellas, puesto que, por definición, este conjunto contiene solamente propiedades que, cuando son poseídas por cualquier cosa, son poseídas esencialmente. Además, la idea es oscura y confusa, porque (a) no es posible que exista algo que posea esencialmente cada una de las propiedades en $M A T_{I}$, y (b) existe al menos una propiedad en $M A T_{I}$ (por ejemplo, tener una forma particular) tal que es posible que exista algo que no la posea necesariamente (p. ej., este pedazo de cera).

Por otra parte, podemos ahora entender porqué Descartes dice que "whenever we call a perception obscure or confused this is because it contains some element of which we are ignorant". ${ }^{62}$ Nótese que 'contiene' en este pasaje debe entenderse como 'contiene objetivamente'. No sólo es que el conjunto $O B J_{I}$ de una idea oscura y confusa $I$ puede contener propiedades que no pertenecen a $M A T_{I}$ (como puede suceder en el caso de algunas ideas claras y distintas), sino que, además, algunas de las propiedades en $M A T_{I}$, si no es que todas, son accidentales y, por tanto, (a) $M A T_{I}$ puede engañarnos con respecto a la esencia verdadera contenida en $O B J_{I}$ (si es que contiene alguna), y (b) es posible que todas las propiedades esenciales en $O B J_{I}$ (si hay tales) estén ocultas, cuando $M A T_{I}$ contiene solamente propiedades no esenciales. Como veremos más adelante, esto es lo que origina la falsedad material:

All self-contradictoriness or impossibility [... arises] when we make the mistake of joining together mutually inconsistent ideas; [...] Self-contradictoriness in our concepts arises merely from their obscurity and confusion..$^{63}$

A fin de explicar esta afirmación, examinemos ahora la falsedad material.

(D) Ideas materialmente falsas

Si $M A T_{I}$ contiene las propiedades $P_{1}, \ldots, P_{m}$ (y ninguna otra), entonces la idea I es materialmente falsa si y solo si no es posible que exista algo que tenga todas las propiedades en $M A T_{I}$; es decir,

$$
\sim \diamond(\exists x)\left(P_{1} x \& \ldots \& P_{m} x\right)
$$

En este caso, $O B J_{I}$ es el conjunto vacío y $M A T_{I}$ no es un subconjunto de $O B J_{I}$. Además, nótese que, como mi análisis predice, una idea que es materialmente falsa tiene que ser oscura y confusa; pero lo contrario no es necesario. ${ }^{64}$

62 VII 147, CSM II 105. Véase también: AT VII 43-44, CSM II 30.

63 AT VII 152, CSM II 108.

64 Véanse AT III 215, CSMK 155; AT VII 164, CSM II 116; y AT VII 233-234, CSM II 163. 
Debemos subrayar que, al contrario que Norman Wells y otros autores, una idea materialmente falsa es una idea: es tanquam rerum, parece representar algo, puesto que su conjunto MAT tiene algún contenido. ${ }^{65}$

Ahora bien, las ideas materialmente falsas carecen de realidad objetiva, ya que la realidad objetiva de una idea es una función de la esencia contenida objetivamente en la idea. ${ }^{66} \mathrm{Sin}$ embargo, las ideas materialmente falsas no contienen objetivamente ninguna esencia; ellas "no representan nada que es real".67 Dado que estas ideas carecen de realidad objetiva, y dado que, de acuerdo con Descartes, la causa de una idea (considerada objetivamente) debe de tener de menos el mismo grado de realidad formal o actual que el grado de realidad objetiva que la idea contiene, ${ }^{68}$ se sigue que las ideas materialmente falsas podrían surgir de un defecto o carencia de perfección en nuestra naturaleza. Descartes afirma algo más fuerte:

I know by the natural light that [...] they are in me only because of a deficiency and lack of perfection in my nature. ${ }^{69}$

En otras palabras, una idea materialmente falsa, considerada objetivamente, puede ser explicada simplemente por el hecho de que, en la formación de su conjunto MAT, la facultad para la formación de ideas, FORM, no fue usada correctamente; en su nivel MAT, la idea viola la cláusula más importante en la definición de esta función, ${ }^{70}$ a saber, que debe de ser posible que exista algo que posea todas las propiedades que están en el conjunto MAT. Que la facultad FORM no haya sido usada es lo que explica por qué

65 Véase la definición de 'idea' en la sección Il y la n. 8 de este artículo. Por el contrario, la posición de Norman Wells en "Material Falsity", p. 37, es que las ideas materialmente falsas son "pseudo-representations, that is, ideas which fail to represent something as it is".

66 Véase AT V 160, CSMK 343.

67 "Nihil reale exhibere." AT VII 234, CSM II 163, traducción mía.

68 Véase AT VI 41, CSM II 28-29. No debe confundirse el concepto de idea considerada formalmente con el concepto de realidad formal. La realidad formal (o actual) de cualquier cosa existente (ya sea una sustancia o un modo) es la perfección que esta cosa tiene en virtud de su esencia (o forma); es decir, en virtud de sus propiedades esenciales: Véase AT V 160, CSMK 343, en donde Descartes dice que una cosa tiene realidad formal en virtud de su esencia eterna e inmutable; y AT V 164, CB [36], 24, en donde sugiere que no existe una distinción real, sino solamente conceptual, entre la esencia y la existencia de una cosa. Por tanto, se sigue que la realidad formal de una cosa, y su existencia, son distinguibles sólo de manera conceptual. En consecuencia, la realidad formal de cualquier cosa, incluso de mis ideas que son modos, es el grado de perfección que esta cosa tiene en tanto que es una cosa existente. Asi considerada, una de mis ideas es simplemente una modificación de mi mente, y es menos perfecta que cualquier otra sustancia existente. Véase AT VII 165, CSM II 117. Por otra parte, la idea considerada formalmente es la idea considerada como una representación o en tanto que representa. Véase AT VII 232, CSM II 163.

69 AT VII 44, CSM II 30. Véanse también: AT VII 46, CSM II 31; y AT VI 38, CSM I 130.

70 Véase AT III 434, CSMK 197. 
la idea, considerada objetivamente, no contiene ninguna esencia y carece de realidad objetiva. ${ }^{71}$

Ahora bien, habíamos visto que Descartes afirmaba que la auto-contradictoriedad o imposibilidad en algunas de nuestras ideas - a saber, aquellas que son materialmente falsas- surge de la oscuridad y confusión de nuestras ideas. ${ }^{72}$ Descartes afirma no sólo que todas las ideas materialmente falsas son oscuras y confusas, sino además que, a veces, erróneamente unimos dos ideas que son, de hecho, mutuamente inconsistentes; ${ }^{73}$ y que esto es posible porque las ideas en cuestión contienen -implícitamente- algún elemento o propiedad esencial que ignoramos.

Consideremos, por ejemplo, la idea escolástica aristotélica de la gravedad, o la pesadez; $;^{74}$ es decir, la cualidad que se pensaba que ciertos cuerpos tenían en virtud de la cual eran acarreados hacia el centro de la Tierra. Esta idea es materialmente falsa (por ende, oscura y confusa) puesto que, aunque la gravedad era concebida como una cualidad "que inhería en los cuerpos sólidos", ${ }^{75}$ la idea también "había sido tomada, en gran medida, de la idea que tenía de la mente". ${ }^{76}$ Es decir, que esta idea involucraba la tesis de que

gravity carried bodies towards the centre of the earth as if it had some knowledge of the centre within itself. For this surely could not happen without knowledge, and there can be no knowledge except in a mind. Nevertheless I continued to apply to gravity various other attributes which cannot be understood to apply to a mind in this way-such as its being divisible, measurable, and so on. ${ }^{77}$

Esta idea de la gravedad - y también las ideas sensoriales de las cualidades sensibles- ${ }^{78}$ es materialmente falsa en el sentido de que no es posible que exista algo que tenga al mismo tiempo modos de pensar y modos de extensión —es decir, propiedades físicas y mentales. En el caso de la idea de gravedad, ésta es materialmente falsa puesto que no es posible que los objetos físicos tengan conocimiento de nada. Uno puede llegar a tener ideas

71 Véase n. 60

72 Véase n. 63.

73 Dos ideas I y $\mathrm{J}$ son, de hecho, mutuamente inconsistentes cuando no es posible que exista algo que posea tanto todas las propiedades contenidas en $O B J_{l}$ como todas las propiedades contenidas en $O B J_{J}$.

74 Véase CSM II 297, n. 1.

75 AT VI 441, CSM II 297, traducción mía.

76 AT VII 442, CSM II 298, traducción mía.

77 Ibid., el subrayado es mío. Véase también: AT III 213, CSMK 155.

78 Aquí no podré defender esta afirmación, debido a los límites de espacio inherentes a cualquier artículo. Véase mi "Descartes on Mental Representation", sección VIIl; y mi artículo "Descartes on Sensible Qualities", (manuscrito). 
falsas como ésas, dice Descartes, cuando uno carece de las ideas claras y distintas de la sustancia corporal y de la sustancia pensante: estas ideas deben incluir (en su nivel MAT) la noción de su distinción real y numérica. ${ }^{79}$ Por el contrario, las ideas oscuras y confusas de estas sustancias pueden muy fácilmente generar ideas falsas, como la idea escolástica de la gravedad, ya que es posible que sólo incluyan, en el nivel MAT, propiedades accidentales, en cuyo caso, las propiedades esenciales, que están necesariamente asociadas a aquéllas, están ocultas y uno puede fácilmente cometer un error con respecto a la distinción real entre la sustancia extensa y la sustancia corporal. En consecuencia, la imposibilidad surge en nuestras ideas cuando unimos dos ideas que son, de hecho, mutuamente inconsistentes, pero cuya inconsistencia no es inmediatamente aparente debido a la oscuridad y confusión de algunas de nuestras ideas.

Finalmente, podemos ahora entender en qué sentido Descartes dice que

it often happens in the case of obscure and confused ideas [...] that an idea is referred to something other than that of which it is in fact the idea. ${ }^{80}$

Descartes no está afirmando que esto es lo que sucede en todos los casos de ideas oscuras y confusas: Una idea oscura y confusa que no es materialmente falsa ${ }^{81}$ es aquella cuyo conjunto MAT contiene propiedades no esenciales que, sin embargo, pueden ser todas poseídas por algo; y no creo que en este caso pueda afirmarse que la idea refiere a algo diferente de aquello a lo que se conforma. Pero, en un cierto sentido, esto sí puede afirmarse de una idea materialmente falsa: A un sujeto descuidado puede parecerle que su conjunto $M A T$ indica algo real, una res que puede existir, cuando de hecho no representa tal cosa.

\section{El contenido objetivo y el genio maligno}

Margaret Wilson piensa que la distinción entre aquello que una idea parece representar y aquello que la idea realmente representa, es desastrosa para Descartes, puesto que, en la tercera Meditación, el argumento de la existencia de Dios requiere que estemos en la posición de saber que es lo que la idea de Dios representa. Sin embargo, una distinción entre lo que una idea parece representar y lo que representa, supuestamente interpondría el velo de la ilusión sobre el objeto representado por la idea. Pero esto sería desastroso, ya que implicaría que podemos estar equivocados acerca de

79 Por ejemplo, la idea clara y distinta de un cuerpo debe incluir (nivel MAT) la noción de que éste es necesariamente una cosa extensa e impensante. Véase AT VII 78, CSM II 54.

80 AT VII 233, CSM II 163, el subrayado es mío.

81 De acuerdo con Descartes, no todas las ideas oscuras y confusas son materialmente falsas. Véase n. 64 . 
este objeto, el cual estaría sujeto a algunas de las dudas sistemáticas de la primera Meditación -como la que genera el imaginario genio maligno de Descartes.

Pero esta distinción, tal y como Descartes la entiende, no tiene esta consecuencia desastrosa. Como hemos visto, el contenido objetivo OBJ (o contenido implícito) de una idea está constituido por la aplicación consistente e infalible de una facultad que nosotros tenemos (la que hemos llamado, la 'facultad FORM'). Cuando la usamos correctamente, nuestras ideas son claras y distintas, y el contenido implícito de las ideas nos es, en principio, accesible. El genio maligno no puede entrar en esta área -como lo hace en la física o la matemática- puesto que lo que está representado por nuestras ideas (el contenido OBJ) depende exclusivamente de una facultad que nosotros poseemos; es decir, depende de un factor que es estrictamente intramental.

Otra cosa es la cuestión de si tal facultad nos da acceso a las esencias inmutables y eternas que, de hecho, Dios decidió crear. ${ }^{82}$ Esto depende de un factor adicional; a saber, que Dios no puede engañarnos ${ }^{83}$ y que, por lo tanto, tuvo que decretar que todas nuestras ideas claras y distintas son verdaderas. ${ }^{84}$

82 Sobre la doctrina de la creación divina de las esencias eternas e inmutables, véanse AT I 152, CSMK 25; y AT IV 118-119, CSMK 235.

83 Véase AT V 147, CSMK 334.

84 Véanse AT III 544-545, CSMK 211; AT III 215, CSMK 155; AT VII 163, CSM II 115; y AT VII 115-117, CSM II 83. 